\title{
A escrita do problema e sua resolução: o entendimento intuitivo acerca da combinatória $^{1}$
}

\section{The written text of mathematical word problems and the success of solution: the intuitive understanding of combinatorics}

\author{
Jane Correa ${ }^{2}$ \\ Gisele Oliveira $^{3}$
}

\section{RESUMO}

Este trabalho pretende examinar a relação entre a escrita e a resolução de problemas envolvendo a noção de combinatória. Foram analisadas a dificuldade relativa que os problemas apresentariam para os estudantes e a influência que a descrição dos valores das variáveis no enunciado do problema traria para sua resolução. Foram apresentadas a 279 estudantes do 5. ${ }^{\circ}$ ao 9..$^{\circ}$ ano do Ensino Fundamental listas com cinco problemas diferentes envolvendo o raciocínio de combinatória (produto cartesiano, combinação, arranjo e permutação) para serem resolvidas individualmente em sala de aula. Estes problemas foram apresentados sob duas formas: a) incluindo a descrição dos valores das variáveis descritas na situação narrada no problema; e b) com o enunciado tradicional, constando apenas o número de valores das variáveis. A modificação na escrita do texto dos problemas por meio da especificação dos valores das variáveis da situação-problema influenciou o sucesso da resolução dependendo da noção de combinatória envolvida no enredo do problema. A variação no enunciado não produziu

1 Nossos agradecimentos ao CNPq (Conselho nacional de desenvolvimento científico e tecnológico) pelo apoio concedido, às crianças e jovens pelo muito que nos ensinaram e aos seus professores pela confiança em nosso trabalho.

2 Doutorado em Psicologia. Professora do Instituto de Psicologia da Universidade Federal do Rio de Janeiro, Brasil.

3 Graduação em Psicologia na Universidade Federal do Rio de Janeiro, Brasil. Bolsista de Iniciação Científica CNPq (Conselho nacional de desenvolvimento científico e tecnológico). 
qualquer efeito facilitador para problemas considerados muito fáceis (produto de medidas) ou difícil demais para os estudantes (permutação). No caso dos problemas combinação e arranjo, cuja resolução foi influenciada pela escrita do enunciado, a declaração dos valores das variáveis no texto do problema pode se tornar uma estratégia didática produtiva para o desenvolvimento do raciocínio combinatório em crianças e adolescentes. Palavras-chave: resolução de problemas; raciocínio multiplicativo; raciocínio combinatório.

\begin{abstract}
This paper intends to examine the relationship between writing and solving combinatorial problems. We analyzed the relative difficulty of various combinatorial problems and the influence that the description of the variable values in the text of the problems would have on their resolution. Two hundred and seventy nine $5^{\text {th }}$ to $9^{\text {th }}$ graders were presented with five different combinatorial problems (Cartesian product, combination, arrangement and permutation) to be solved individually in the classroom. These problems were presented in two forms: a) including the description of the variable values in the text of the problems and $b$ ) the traditional combinatorial word problem. The change in the text of the problems influenced the success of their resolution depending on the combinatorial concept involved in the word problem. The variation in the text problem had no effect on the resolution of problems considered too easy (Cartesian product) or too difficult (permutation) by the students. In the case of the combination and arrangement problems, whose resolution was influenced by the change in the problem text, the declaration of the variable values in the text of the problem might become a productive teaching strategy for the development of combinatorial reasoning for children and adolescents.

Keywords: problems resolution; multiplicative reasoning; combinatorial reasoning.
\end{abstract}




\section{A Contagem como Operação Matemática Fundamental e a Noção de Combinatória}

A contagem é uma operação matemática básica e fundamental cujo desenvolvimento leva à construção do conceito de número, do sistema de numeração e das operações matemáticas (PIAGET; SZEMINSKA, 1975). O desenvolvimento da contagem na criança fundamenta-se inicialmente no esquema de correspondência (PIAGET; SZEMINSKA, 1975). Pelo emprego da correspondência, as crianças mais novas, sem a necessidade de enumerar os elementos, podem julgar a equivalência de quantidades, bem como comparar coleções fazendo julgamentos a partir do uso de códigos relativos - mais ou menos que, maior ou menor que (NUNES; BRYANT, 1991). Por volta dos 6-7 anos, a criança é capaz de, com certa facilidade, realizar comparações entre coleções pela enumeração dos seus elementos (FAYOL, 1990). Ao contar, a criança mostra, então, o entendimento em ato de uma série de princípios, dentre os quais: a) o da correspondência termo a termo estrita entre os objetos contados e o nome dos números; b) o da cardinalidade, c) o da ordem estável do nome dos números, d) o da abstração; e e) o da não pertinência da ordem dos objetos contatos.

O uso da correspondência termo a termo é importante também para a construção das operações aditivas bem como contribui para a construção das primeiras noções acerca da multiplicação, baseada na repetição de agrupamentos de mesma quantidade, e da divisão, a partir do entendimento da equivalência e do uso do esquema de distribuição (CORREA; NUNES; BRYANT, 1998; NUNES; BRYANT, 1997). Entretanto, o entendimento da multiplicação e divisão, como operações de natureza multiplicativa, vai impor à criança o domínio não mais da correspondência termo a termo, mas da correspondência um para muitos (PIAGET, 1977).

Diferentemente da adição e da subtração, operações utilizadas na solução de situações-problema envolvendo relações parte-parte e parte-todo, a multiplicação e a divisão são empregadas na modelagem de problemas envolvendo dois ou mais fatores considerados simultaneamente. Desta forma, a solução das situações-problema de estrutura multiplicativa envolve não mais as ações de juntar (somar) e retirar (subtrair) como nos problemas de natureza aditiva, mas o produto das ações realizadas simultaneamente (NUNES; BRYANT, 1997; VERGNAUD, 1979). Assim sendo, os problemas de estrutura multiplicativa impõem uma complexidade maior para sua solução do que os problemas de estrutura aditiva (VERGNAUD, 1990).

Enquanto os problemas de estrutura aditiva podem ser classificados em 
três grandes classes - transformação, combinação, comparação (CARPENTER; MOSER, 1983, VERGNAUD, 1979; 1990), os problemas de estrutura multiplicativa podem ser agrupados em um número muito maior de classes e, consequentemente, respondendo por uma quantidade maior de situações e sentidos (GREER, 1992; VERGANUD, 1979; 1990). As situações-problema de estrutura multiplicativa mais frequentes no ensino fundamental são aquelas relacionadas à formação de agrupamentos equivalentes.

Existe, no entanto, outra classe de problemas de estrutura multiplicativa que aparece com relativa frequência nas primeiras séries escolares: aquela que envolve o princípio fundamental de contagem sob a forma de produto cartesiano (MORO; SOARES, 2006). Tais situações-problema são apresentadas nos anos iniciais do ensino fundamental frequentemente sob a forma de tabela de dupla entrada a partir da qual as crianças realizam a correspondência entre os elementos e após contam a quantidade de pares formados.

A compreensão do princípio fundamental de contagem fornece o fundamento para o entendimento dos problemas de análise combinatória, ou seja, situações-problema envolvendo a permutação, o arranjo e a combinação. A análise combinatória compreende métodos que permitem a contagem de agrupamentos formados em determinadas condições. Problemas de combinatória simples podem ser resolvidos pelo princípio fundamental de contagem, entretanto, se o número de agrupamentos a serem contados for grande, é necessário o conhecimento das técnicas de contagem da análise combinatória para a solução da tarefa.

O entendimento das diferentes técnicas de contagem da análise combinatória traz bastante dificuldade para o aprendiz tanto no que se refere ao domínio dos algoritmos bem como o de seu uso nas situações-problema que modelam (BATANERO; NAVARRO-PELAYO; GODINO, 1997; TAXA-AMARO, 2006). O emprego de qualquer das técnicas de contagem da análise combinatória exige em primeiro lugar a compreensão dos diversos modos de formar os agrupamentos, se por arranjo, permutação ou combinação (SODRÉ, 2010). Nas situações-problema envolvendo a noção de arranjo, os agrupamentos são formados com elementos distintos entre si pela ordem ou pela espécie (por exemplo, AB e BA são duas ocorrências distintas); na permutação, os agrupamentos são distintos pela ordem com que todos os elementos são dispostos e, finalmente, na combinação, os agrupamentos são distintos entre si apenas pela espécie (por exemplo, AB e BA produzem o mesmo resultado).

Se a resolução de problemas de análise combinatória torna-se praticamente impossível sem o emprego de um algoritmo de contagem, situações-problema envolvendo pequenos números podem ser resolvidas, no entanto, prescindindo-se do uso de tais métodos (PIAGET; INHELDER, 1951). Tal fato, aliado à variedade 
de domínios nos quais a combinatória poderia ser aplicada e às diferentes habilidades que poderiam ser desenvolvidas a partir dos problemas de combinatória (contagem, numeração, multiplicação, divisão, probabilidade, entre outras), faz com que a apresentação de situações didáticas envolvendo noções elementares de combinatória possa fazer parte do currículo escolar desde as primeiras séries do ensino fundamental (ENGLISH, 1994).

\section{A Escrita do Problema e sua Resolução}

A resolução de problemas bem como o desenvolvimento de procedimentos para sua solução são aspectos importantes da atividade matemática (BRITO; CORREA, 2004). Uma vez que os problemas em matemática são apresentados aos alunos sob forma de um texto escrito, sua resolução depende não apenas do domínio de conceitos matemáticos por parte dos alunos, mas também da interpretação do enunciado do problema, ou seja, do seu texto. É a partir da leitura do texto escrito que a criança construirá seu entendimento da natureza matemática do problema e extrairá as informações matemáticas de que precisa para traduzir as situações apresentadas no texto em uma expressão matemática.

A compreensão de textos é uma atividade complexa influenciada por diversos fatores, entre os quais, o conhecimento de mundo e o domínio do gênero de texto a ser lido. Desta forma, o sucesso na compreensão de texto não recai apenas na habilidade verbal geral, sendo também relacionado a aspectos mais contingentes com referência à natureza do texto lido e de seu conteúdo. Os problemas verbais matemáticos, como gênero textual, vão requerer o entendimento de sua estrutura e de um roteiro (script) a ser seguido.

Um problema verbal matemático é composto de um enunciado (onde é descrita a situação-problema) e uma pergunta. O roteiro a ser seguido inclui a tradução do enunciado verbal do problema em representação matemática, a execução de procedimentos para a sua solução e a redação da resposta do problema a partir da informação matemática encontrada.

Embora o uso de situações-problema seja importante para examinar a compreensão de conceitos matemáticos, uma vez que tais situações dão sentido às noções aprendidas, a avaliação do conhecimento matemático, expresso em sua execução, não pode ser desvinculada da análise do enunciado do próprio problema e de sua estrutura (BRITO; CORREA, 2004).

Nesse sentido, pretendemos examinar os primórdios do entendimento do princípio fundamental de contagem e das noções de permutação, arranjo e 
combinação, analisando a influência da escrita do problema no seu entendimento. Particularmente, interessa-nos o exame da compreensão dos problemas de estrutura multiplicativa que envolvem o princípio fundamental de contagem e o raciocínio combinatório levando-se em conta variações no texto do problema relacionadas à descrição dos valores de cada variável, isto é, dos elementos colocados em correspondência.

\section{Método}

Participaram desta pesquisa 279 estudantes, de ambos os sexos, do Ensino Fundamental de colégio público da cidade do Rio de Janeiro, sendo: 46 do quinto ano, 60 do sexto ano, 55 do sétimo ano, 61 do oitavo ano e 57 do nono ano. Quatro problemas envolvendo o raciocínio combinatório (produto cartesiano, combinação, arranjo e permutação) foram organizados em duas listas de acordo com o tipo de enunciado, sendo um enunciado prototípico dos problemas do gênero e outro com a especificação dos valores das variáveis conforme mostra o Quadro I. Metade das crianças em cada ano escolar resolveu a lista de problemas com enunciado tradicional e a outra metade a lista de problemas cujo enunciado incluía a especificação dos valores das variáveis.

\begin{tabular}{|c|c|c|}
\hline & Especificação dos fatores & Enredo tradicional \\
\hline $\begin{array}{l}\text { Produto } \\
\text { Cartesino }\end{array}$ & $\begin{array}{l}\text { Uma moça possui } 4 \text { blusas de cores } \\
\text { diferentes (azul, verde, preta e branca) } \\
\text { e } 3 \text { modelos de saia (lisa, listrada } \\
\text { e bordada). De quantas maneiras } \\
\text { diferentes ela pode vestir uma blusa e } \\
\text { uma saia? }\end{array}$ & $\begin{array}{l}\text { Uma moça possui } 4 \text { blusas de cores diferentes } \\
\text { e } 3 \text { modelos de saia. De quantas maneiras } \\
\text { diferentes ela pode vestir uma blusa e uma } \\
\text { saia? }\end{array}$ \\
\hline Combinação & $\begin{array}{l}\text { Uma lanchonete tem } 4 \text { tipos de frutas } \\
\text { (banana, laranja, uva e maçã) para fazer } \\
\text { uma minissalada. Cada minissalada } \\
\text { é composta de } 3 \text { frutas diferentes. De } \\
\text { quantas maneiras diferentes as saladas } \\
\text { podem ser preparadas? }\end{array}$ & $\begin{array}{l}\text { Uma lanchonete tem } 4 \text { tipos de frutas para fazer } \\
\text { uma minissalada. Cada minissalada é composta } \\
\text { de } 3 \text { frutas diferentes. De quantas maneiras } \\
\text { diferentes as saladas podem ser preparadas? }\end{array}$ \\
\hline
\end{tabular}




\begin{tabular}{|l|l|l|}
\hline Arranjo & $\begin{array}{l}\text { Em um concurso para modelo existem } \\
\text { 4 candidatas (Maria, Joana, Carla } \\
\text { e Antonia). De quantas maneiras } \\
\text { diferentes podemos ter o resultado do } \\
\text { concurso premiando o primeiro e o } \\
\text { Permutação }\end{array}$ & $\begin{array}{l}\text { Em um concurso para modelo existem 4 } \\
\text { candidatas. De quantas maneiras diferentes } \\
\text { podemos ter o resultado do concurso } \\
\text { premiando o primeiro e o segundo lugar? }\end{array}$ \\
& $\begin{array}{l}\text { Quatro pessoas (João, Maria, Carlos e } \\
\text { Pentadas em uma fileira de cadeiras } \\
\text { uma ao lado da outra. De quantas } \\
\text { maneiras diferentes as pessoas podem } \\
\text { se arrumar uma ao lado da outra para } \\
\text { a foto? }\end{array}$ & $\begin{array}{l}\text { Quatro pessoas vão posar para uma fotografia } \\
\text { sentadas em uma fileira de cadeiras uma ao a do outra. De quantas maneiras diferentes } \\
\text { lado do para a foto? }\end{array}$ \\
\hline
\end{tabular}

QUADRO 1 - VARIAÇÃO NO TEXTO DOS DIVERSOS PROBLEMAS ENVOLVENDO A NOÇÃO DE COMBINATÓRIA

As listas de problemas foram apresentadas nas salas de aula de cada turma pelo pesquisador, sendo solicitado que aos estudantes que solucionassem os problemas da melhor forma, podendo usar qualquer tipo de procedimento e notação que achassem convenientes para a resolução da situação-problema apresentada. Em cada lista, os problemas estavam dispostos um em cada página, havendo respectivamente o enunciado, um espaço para resolução, outro para a resposta e, finalmente, um espaço no qual o aprendiz poderia explicar como pensou para resolver o problema.

\section{Resultados}

A análise dos resultados será apresentada em duas etapas. Analisamos inicialmente a dificuldade relativa dos problemas de combinatória em função da escolaridade dos aprendizes. Em seguida, examinamos se as variações no enunciado do problema auxiliaram os aprendizes na resolução dos diferentes tipos de problemas de combinatória.

1. A dificuldade relativa dos diferentes problemas envolvendo a combinatória segundo os aprendizes

A Tabela 1 apresenta a porcentagem de acertos na resolução dos diversos problemas em função da escolaridade dos estudantes. A distribuição de tais percentagens revela que, de forma geral, o desempenho dos estudantes varia significativamente de acordo com o tipo de problema apresentado $\left(\mathrm{F}_{\text {Greenhouse-Geisser }}\right.$ 
$(2,7774)=224,28 ; \mathrm{p}<.001)$. Em ordem crescente de dificuldade: produto de medidas (o de mais fácil resolução), combinação, arranjo e permutação (problema com o menor índice de acertos). Tais resultados são mais bem entendidos se examinadas as interações que se estabelecem entre a escolaridade e a noção de combinatória a que a situação-problema se refere $\left(\mathrm{F}_{\text {Greenhouse-Geisser }(10,81 ; 741)}=3,32 ; \mathrm{p}<.001\right)$.

\begin{tabular}{|c|c|c|c|c|c|c|c|c|}
\hline \multirow[b]{2}{*}{ ANO } & \multicolumn{4}{|c|}{ Enunciado Tradicional } & \multicolumn{4}{|c|}{$\begin{array}{l}\text { Enunciado com Especificação dos Valores das } \\
\text { Variáveis }\end{array}$} \\
\hline & $\begin{array}{c}\text { Produto } \\
\text { de } \\
\text { Medidas }\end{array}$ & Combinação & Arranjo & Permutação & $\begin{array}{l}\text { Produto } \\
\text { de } \\
\text { Medidas }\end{array}$ & Combinação & Arranjo & Permutação \\
\hline $\begin{array}{l}5 \\
(n=46)\end{array}$ & 52 & 13 & 4 & 0 & 61 & 39 & 30 & 9 \\
\hline $\begin{array}{l}6 \\
(\mathrm{n}=60)\end{array}$ & 93 & 37 & 23 & 13 & 90 & 30 & 20 & 13 \\
\hline $\begin{array}{l}7 \\
(\mathrm{n}=55)\end{array}$ & 100 & 25 & 7 & 7 & 100 & 56 & 22 & 15 \\
\hline $\begin{array}{l}8 \\
(n=61)\end{array}$ & 96 & 15 & 19 & 15 & 97 & 40 & 49 & 26 \\
\hline $\begin{array}{l}9 \\
(\mathrm{n}=57)\end{array}$ & 97 & 41 & 37 & 34 & 100 & 68 & 64 & 20 \\
\hline
\end{tabular}

Problemas de produto cartesiano não parecem ser bem compreendidos antes do $6 .^{\circ}$ ano. No $5 .^{\circ}$ ano observa-se uma percentagem média de acertos em torno de $57 \%$, enquanto no $6 .^{\circ}$ ano as resoluções corretas atingem uma percentagem média, em termos gerais, de $92 \%$, praticamente um efeito de teto. No entanto, outros problemas continuam de difícil resolução mesmo para aqueles que cursavam o último ano do ensino fundamental. Em média, cerca de $55 \%$ dos estudantes do $9 .^{\circ}$ ano obtiveram sucesso na resolução do problema envolvendo a noção de combinação e $51 \%$ no problema de arranjo. Em contraste, apenas um quarto dos alunos do $9 .^{\circ}$ ano foi capaz de resolver o problema que incluiu a ideia de permutação.

Para agruparmos os estudantes em função do seu desempenho individual na realização dos diversos problemas, foi realizada uma análise hierárquica de aglomerados. Esta análise resultou na distribuição dos estudantes em quatros grupos, conforme mostra a Tabela 2. 
TABELA 2 - PERCENTAGEM DE RESOLUÇÕES CORRETAS PARA OS DIVERSOS PROBLEMAS DE COMBINATÓRIA SEGUNDO OS GRUPOS FORMADOS PELAANÁLISE DE AGLOMERADOS

\begin{tabular}{lcccc}
\hline $\begin{array}{l}\text { Grupo I } \\
(\mathrm{n}=174)\end{array}$ & produto de medidas & combinacão & arranjo & permutacão \\
\cline { 2 - 5 } & 100 & 32 & 00 & 07 \\
$\begin{array}{l}\text { Grupo II } \\
(\mathrm{n}=28)\end{array}$ & 00 & 18 & 07 & 00 \\
$\begin{array}{l}\text { Grupo III } \\
(\mathrm{n}=35)\end{array}$ & 100 & 00 & 100 & 46 \\
$\begin{array}{l}\text { Grupo IV } \\
(\mathrm{n}=42)\end{array}$ & 100 & 100 & 100 & 40 \\
\hline
\end{tabular}

No Grupo I encontram-se os alunos que, em sua maioria, foram bemsucedidos na resolução do problema de produtos de medidas. No Grupo II, os estudantes com baixo desempenho nos diversos problemas. Os Grupos III e IV se distinguem pelo desempenho de seus membros nos problemas de combinação. No Grupo III estão os alunos que não conseguiram resolver o problema de combinação, tendo solucionado, no entanto, os problemas de produto de medidas e arranjo. No Grupo IV, por sua vez, estão os estudantes com bom desempenho nos problemas de produto de medidas, combinatória e arranjo. Nos Grupos I e II os alunos demonstram não fazer sentido da situação-problema de permutação. Nos Grupos III e IV observa-se um percentual de acertos para o problema de permutação em torno de $40 \%$. A Tabela 3 apresenta a distribuição dos estudantes por estes agrupamentos segundo sua escolaridade.

TABELA 3 - DISTRIBUIÇÃO EM PORCENTAGEM DOS ESTUDANTES POR GRUPOS SEGUNDO A ESCOLARIDADE

\begin{tabular}{llllll}
\hline & \multicolumn{5}{c}{ Escolaridade } \\
\hline & 5 & 6 & 7 & 8 & 9 \\
\hline $\begin{array}{l}\text { Grupo I } \\
(\mathrm{n}=174)\end{array}$ & 42 & 70 & 85 & 62 & 49 \\
\hline $\begin{array}{l}\text { Grupo II } \\
(\mathrm{n}=28)\end{array}$ & 43 & 8 & 0 & 4 & 2 \\
\hline $\begin{array}{l}\text { Grupo III } \\
(\mathrm{n}=35)\end{array}$ & 4 & 12 & 9 & 16 & 19 \\
$\begin{array}{l}\mathrm{G}=350 \text { IV } \\
(\mathrm{n}=42)\end{array}$ & 11 & 10 & 6 & 18 & 30 \\
\hline
\end{tabular}

O Grupo I inclui a maioria dos estudantes do $6 .^{\circ}$ ao $9 .^{\circ}$ anos. Os alunos do 5. ${ }^{\circ}$ ano, por outro lado, distribuem-se equitativamente entre os Grupos I e II. Os alunos dos $8 .^{\circ}$ e $9 .^{\circ}$ anos, embora, a exemplo dos alunos dos outros anos, estejam 
em maioria no Grupo I, têm percentagem expressiva de estudantes (34\% para o $8 .^{\circ}$ ano e $49 \%$ para o $9 .^{\circ}$ ano) distribuída pelos Grupos III e IV.

\section{A escrita do problema e sua resolução}

À exceção do $6 .^{\circ}$ ano, as variações produzidas na escrita do enunciado do problema auxiliaram o entendimento de situações-problema relacionadas à combinação e arranjo conforme demonstra a Tabela 1. O mesmo efeito, porém, não foi observado em relação aos problemas de produto cartesiano e de permutação. Em outras palavras, declarar os valores das variáveis no enunciado dos problemas não teve qualquer efeito na resolução dos estudantes quando as situações-problema envolviam por um lado a mais fácil das situações-problema (produto cartesiano) ou, por outro lado, a mais difícil (permutação).

A análise qualitativa dos procedimentos de resolução dos problemas de combinação e de arranjo, ainda que exploratória, auxiliaria a compreensão da facilitação exercida pela declaração dos valores das variáveis no enunciado do problema para o desempenho dos estudantes (Tabelas 4 e 5).

TABELA 4 - DISTRIBUIÇÃO DOS PROCEDIMENTOS DE RESOLUÇÃO PARA O PROBLEMA DE COMBINAÇÃO (EM PORCENTAGEM)

\begin{tabular}{lllll}
\hline \multirow{2}{*}{ enunciado } & tradicional & enumeração & multiplicação & outros \\
\cline { 3 - 5 } & declaração dos valores das & 91 & 3 & 5 \\
& variáveis & & & 6 \\
& & & & \\
\hline Total & 92 & 2 & 6 \\
\hline
\end{tabular}

TABELA 5 - DISTRIBUIÇÃO DOS PROCEDIMENTOS DE RESOLUÇÃO PARA O PROBLEMA DE ARRANJO (EM PORCENTAGEM)

\begin{tabular}{lllll} 
& & enumeração & contagem & multiplicação \\
\cline { 3 - 5 } enunciado & tradicional & 74 & 16 & 10 \\
& declaração dos valores das & 76 & 24 & \\
& variáveis & & &
\end{tabular}

Total 
Foram observados alguns poucos procedimentos que respondem pela maioria das resoluções dos problemas envolvendo noções de combinação e arranjo. O procedimento mais empregado foi o da enumeração sistemática de todos os casos possíveis para a situação-problema e a posterior contagem de todos os casos. Tal procedimento foi observado, em geral, para $93 \%$ das resoluções para o problema de combinação e para $75 \%$ para o de arranjo. O emprego do algoritmo da multiplicação, quer por si só, quer seguido de correspondências estabelecidas entre alguns poucos elementos, teve uma ocorrência média de $2 \%$ para o problema de combinação e $4 \%$ para o de arranjo. Por outro lado, no caso do problema envolvendo a noção de arranjo, uma porcentagem expressiva de resoluções, $21 \%$ em média, referiu-se ao princípio de contagem, ou seja, produto dos elementos disponíveis nas diversas etapas, considerando explicitamente para cada uma das etapas o número de elementos da etapa anterior. Não há diferenças significativas quanto à distribuição dos procedimentos de solução segundo o texto escrito no enunciado dos problemas de combinação $\left(\mathrm{X}^{2}=.65, \mathrm{p}=.72\right) \mathrm{e}$ de arranjo $\left(X^{2}=2,88, p=.27\right)$. Desta forma, observa-se que a influência exercida pela modificação no enunciado do problema foi no número de resoluções e não na natureza dos procedimentos empregados.

\section{Discussão}

Examinamos a resolução de problemas envolvendo o raciocínio combinatório por estudantes entre o $5 .^{\circ}$ e $9 .^{\circ}$ anos do ensino fundamental. Foram analisadas a dificuldade relativa que os problemas apresentariam para os estudantes e a influência que a descrição dos valores das variáveis no enunciado do problema traria para sua resolução.

No problema de produto cartesiano ou de produto de medidas a média de acertos foi alta e variou muito pouco do $6 .^{\circ}$ ao $9 .^{\circ}$ ano em relação ao tipo de enunciado apresentado. Mesmo sendo considerada a dificuldade encontrada pelos estudantes do $5 .^{\circ}$ ano, pode-se dizer que o problema de produto de medidas se mostrou o menos complexo dos problemas apresentados aos estudantes.

O desempenho dos estudantes no problema que envolveu a noção de permutação foi, de modo geral, muito baixo, revelando que este tipo de situação-problema se mostrou extremamente complexo para os alunos em todos os anos escolares. A variação na resolução deste problema em relação ao tipo de enunciado apresentado foi inexpressiva. Nos problemas de combinação e de arranjo, por outro lado, os acertos variaram quanto ao tipo de enunciado 
apresentado. Observou-se um número relativamente maior de acertos para ambos os tipos de problemas quando os enunciados incluíam a especificação dos valores das variáveis.

O conjunto dos resultados acima mostra que a modificação na escrita do texto dos problemas por meio da especificação dos valores das variáveis da situação-problema influenciou o sucesso da resolução dependendo da noção de combinatória envolvida no enredo do problema. A variação no enunciado não produziu qualquer efeito facilitador para problemas considerados muito fácil (produto cartesiano) ou difícil demais (permutação) para os estudantes. No caso dos problemas combinação e arranjo, cuja resolução foi influenciada pela escrita do enunciado, a declaração dos valores das variáveis no texto do problema pode se tornar uma estratégia didática produtiva para o desenvolvimento do raciocínio combinatório em crianças e jovens.

A variação no enunciado dos problemas, por outro lado, não resultou no emprego pelos estudantes de procedimentos de resolução diferentes daqueles usados para a resolução dos problemas com enunciado tradicional. A modificação realizada no enunciado dos problemas produziu, no entanto, um número maior de resoluções corretas. Isto significa que um maior número de estudantes pôde representar a situação-problema de forma mais acurada empregando os procedimentos correntes de resolução.

A natureza dos procedimentos de resolução dos problemas de arranjo e combinação ofereceu indícios para a compreensão da dificuldade relativa entre os problemas como para nos auxiliar no entendimento do papel exercido pela modificação realizada no enunciado para alguns problemas e não outros. A enumeração exaustiva dos casos foi a estratégia de resolução mais frequentemente empregada pelos alunos. Em função dos números usados nos problemas, havia respectivamente 4 combinações e 12 arranjos possíveis. Embora o emprego da estratégia fosse facilitado pela presença dos números usados no problema, é a inclusão dos valores das variáveis no enunciado do problema que melhor explica o aumento no número de resoluções para estes problemas. A descrição dos valores das variáveis no enunciado parece ter favorecido estabelecimento de correspondência sistemática dos elementos segundo a noção de combinatória envolvida em cada uma das situações-problema.

O mesmo não foi observado para o problema de permutação, o problema de mais difícil resolução. Ainda sim com os baixos números empregados no problema, no caso da permutação, os casos gerados eram relativamente numerosos (24 casos possíveis) quando comparados com aqueles encontrados para os problemas de combinação e arranjo. O aumento no número de casos possíveis no problema dificultou a sistematicidade da ação de construção dos agrupamentos e o monitoramento da atividade de forma que se controle o grupo 
que já foi realizado.

O estabelecimento de correspondências parciais não exaustivas ou a dificuldade no monitoramento das correspondências já realizadas são obstáculos à resolução dos problemas de raciocínio combinatório (BATANERO; NAVARROPELAYO; GODINO, 1997; GODINO; BATANERO; ROA, 2005; PIAGET; INHELDER, 1951). A declaração dos valores das variáveis favoreceu a que um número expressivo de estudantes pudessem, a partir do conhecimento dos elementos a serem colocados em correspondência e da singularidade de cada correspondência estabelecida, suplantar os obstáculos para a realização da enumeração sistemáticas dos casos possíveis. Porém, a facilitação da escrita do texto sobre a realização da enumeração sistemática dos possíveis foi possível para as operações de combinação e arranjo mas não para permutação. Tal fato nos remete a outro obstáculo epistemológico relacionado à construção do raciocínio combinatório: a compreensão da noção de combinatória envolvida no problema e a consequente diferenciação das diversas ações para formação dos agrupamentos associadas a cada situação-problema (BATANERO; NAVARROPELAYO; GODINO, 1997; GODINO; BATANERO; ROA, 2005).

Nesse sentido, a ideia da permutação permanece uma das mais difíceis no que se refere à construção do raciocínio combinatório (PIAGET; INHELDER, 1951). A permutação envolveria uma operação de segunda ordem, uma vez que se trata não só da realização de trocas de ordem, mas da multiplicação das trocas de ordem. Diferentemente, no caso das noções de combinação e arranjo, importa coordenar duas operações entre si: a seriação e a correspondência (PIAGET; INHELDER, 1951). A distinção entre as duas noções recai, então, na relevância da ordem (BATANERO; NAVARRO-PELAYO; GODINO, 1997). Em outras palavras, em distinguir a ordem dos elementos como relevante para distinção dos casos possíveis quando deveria ser. Neste sentido, em termos das operações realizadas na construção intuitiva do raciocínio combinatório, apesar das dificuldades impostas pelo desenvolvimento do pensamento multiplicativo, torna-se relativamente mais fácil para crianças e jovens a composição multiplicativa das operações de seriação e correspondência de uma única operação do que a realização da multiplicação como operação de segunda ordem.

Promover o desenvolvimento do raciocínio combinatória nos estudantes ao longo do ensino fundamental parece implicar, a nosso ver, na criação de oportunidades que resultem, para os aprendizes, em cognições acerca das diferentes noções relacionadas à combinatória a partir das ações e representações vinculadas à composição multiplicativa dos possíveis e a multiplicação das ordens possíveis. Neste sentido, a declaração dos valores das variáveis no enunciado do problema pode ser um recurso para favorecer a compreensão acerca das operações segundo as quais os casos possíveis serão gerados para 
a situação-problema. O significado atribuído desde o início aos elementos que serão postos, de forma sistemática, em correspondência às composições então geradas, auxiliaria, assim, os estudantes na compreensão e na diferenciação das diversas classes de situações-problema relacionadas ao raciocínio combinatório.

\section{REFERÊNCIAS}

BATANERO, C.; NAVARRO-PELAYO, V.; GODINO, J. D. Effect of the implicit combinatorial model on combinatorial reasoning in secondary school pupils. Educational Studies in Mathematics, Netherlands, v. 32, n. 2, p. 181-199, 1997.

BRITO, M. R. F.; CORREA, J. Divisão e representação no processo de solução de problemas aritméticos. In: PIROLA, N. A.; TAXA-AMARO, F. S. O. (Org.). Educação Matemática. Pedagogia Cidadã. Cadernos do professor. São Paulo: UNESP, 2004. p. 81-90.

CARPENTER, T. P.; MOSER, J. M. The acquisition of addition and subtraction concepts. In: LESH, R.; LANDAU, M. (Eds.). Acquisition of mathematics concepts and processes. London: Academic Press, 1983. (Developmental Psychology Series). p. 7-44.

CORREA, J.; NUNES, T. N.; BRYANT, B. Young children's understanding of division: the relationship between division terms in a non-computational task. Journal of Educational Psychology, v. 90, n. 2, p. 321-329, 1998.

ENGLISH, L. D. Young children's combinatorial strategies. Educational Studies in Mathematics, 22, p. 451-474, 1974.

FAYOL, M. A criança e o número. Porto Alegre: Artes Médicas, 1990.

GREER, B. Multiplication and division as models of situations. In: GROUWS, D. (Ed.). Handbook of research on Mathematics teaching and learning - A project of the National Council of Teachers of Mathematics. New York: Macmillian Library Reference USA, 1992. p. 276-295.

MORO, M. L.; SOARES, M. T. Níveis de raciocínio combinatório e produto cartesiano na escola fundamental. Educação Matemática Pesquisa, v. 8, n. 1, p. 99-124, 2006.

NUNES, T.; BRYANT, P. E. Correspondência: um esquema quantitativo básico. Psicologia: Teoria e Pesquisa, v. 7, n. 3, p. 237-284, 1991. 
. Crianças fazendo matemática. Tradução de: COSTA, S. Porto Alegre: Artes Médicas, 1997.

PIAGET, J. Recherches sur l'abstraction réfléchissante - l'abstraction des relations logico-arithmétiques. Paris: PUF, 1977. Études D’Épistémologie Génetique, v. XXXIV.

PIAGET, J.; INHELDER, B. A origem da ideia de acaso na criança. Tradução de: COELHO, A. M. Rio de Janeiro: Record, 1951.

PIAGET, J.; SZEMINSKA, A. A gênese do número na criança. Tradução de: OITICICA, C. M. Rio de Janeiro: Zahar, 1975.

SODRÉ, U. Ensino médio: análise combinatória. Disponível em: <http://pessoal. sercomtel.com.br/matematica/medio/combinat/combinat.htm>. Acesso em: 28/2/2010.

TAXA-AMARO, F. O. S. Solução de problemas com operações combinatórias. In: BRITO, M. R. F. (Org.). Solução de problemas e a matemática escolar. Campinas: Alínea, 2006. p. 163-183.

VERGNAUD, G. The acquisition of arithmetical concepts. Educational Studies in Mathematics, n. 10, p. 263-274, 1979. . La théorie des champs conceptuels. Recherches en Didactiques des Mathématiques, v. 10, n. 23, p. 133-170, 1990. 
